Annales Academiæ Scientiarum Fennicæ

Mathematica

Volumen 35, 2010, 115-130

\title{
COMPACT EMBEDDINGS FOR SOBOLEV SPACES OF VARIABLE EXPONENTS AND EXISTENCE OF SOLUTIONS FOR NONLINEAR ELLIPTIC PROBLEMS INVOLVING THE $p(x)$-LAPLACIAN AND ITS CRITICAL EXPONENT
}

\author{
Yoshihiro Mizuta, Takao Ohno, Tetsu Shimomura and Naoki Shioji
}

Hiroshima University, Department of Mathematics, Graduate School of Science Higashi-Hiroshima 739-8521, Japan; mizuta@mis.hiroshima-u.ac.jp

Hiroshima National College of Maritime Technology, General Arts

Higashino Oosakikamijima Toyotagun 725-0231, Japan; ohno@hiroshima-cmt.ac.jp

Hiroshima University, Department of Mathematics, Graduate School of Education Higashi-Hiroshima 739-8524, Japan; tshimo@hiroshima-u.ac.jp

Yokohama National University, Department of Mathematics

Tokiwadai, Hodogaya-ku, Yokohama 240-8501, Japan; shioji@math.sci.ynu.ac.jp

Abstract. We give a sufficient condition for the compact embedding from $W_{0}^{k, p(\cdot)}(\Omega)$ to $L^{q(\cdot)}(\Omega)$ in case ess $\inf _{x \in \Omega}(N p(x) /(N-k p(x))-q(x))=0$, where $\Omega$ is a bounded open set in $\mathbf{R}^{N}$. As an application, we find a nontrivial nonnegative weak solution of the nonlinear elliptic equation

$$
-\operatorname{div}\left(|\nabla u(x)|^{p(x)-2} \nabla u(x)\right)=|u(x)|^{q(x)-2} u(x) \quad \text { in } \Omega, \quad u(x)=0 \quad \text { on } \partial \Omega .
$$

We also consider the existence of a weak solution to the problem above even if the embedding is not compact.

\section{Introduction}

In recent years, many authors have studied the generalized Lebesgue spaces; see $[2,5,8-23,26-29,32]$. First, let us recall some definitions. Following Orlicz [29] and Kovácik and Rákosník [22], for an open set $\Omega$ in $\mathbf{R}^{N}$ with $N \geq 1$ and a measurable function $p(\cdot): \Omega \rightarrow[1, \infty)$, we define the $L^{p(\cdot)}(\Omega)$-norm of a measurable function $f$ on $\Omega$ by

$$
\|f\|_{L^{p(\cdot)}(\Omega)}=\inf \left\{\lambda>0: \int_{\Omega}\left|\frac{f(x)}{\lambda}\right|^{p(x)} d x \leq 1\right\}
$$

and denote by $L^{p(\cdot)}(\Omega)$ the family of all measurable functions whose $L^{p(\cdot)}(\Omega)$-norms are finite. Further we denote by $W^{k, p(\cdot)}(\Omega)$ with $k \in \mathbf{N}$ the family of all measurable functions $u$ on $\Omega$ such that

$$
\|u\|_{W^{k, p(\cdot)}(\Omega)}=\sum_{0 \leq|\alpha| \leq k}\left\|D^{\alpha} u\right\|_{L^{p(\cdot)}(\Omega)}<\infty
$$

and by $W_{0}^{k, p(\cdot)}(\Omega)$ the closure of $C_{0}^{\infty}(\Omega)$ in $W^{k, p(\cdot)}(\Omega)$.

doi:10.5186/aasfm.2010.3507

2000 Mathematics Subject Classification: Primary 35J20, 46B50, 46 E30.

Key words: Sobolev spaces of variable exponents, compact embeddings, nonlinear elliptic problems.

The fourth author was supported in part by Grant-in-Aid for Scientific Research (C) (No. 17540149) Japan Society for Promotion of Science. 
Recently, Kurata and the fourth author [23] posed the following problem: if a variable exponent $q(\cdot)$ satisfies $2<\operatorname{essinf}_{x \in \Omega} q(x) \leq \operatorname{ess}_{\sup } \in \Omega q(x) \leq 2 N /(N-2)$ $(N \geq 3)$ and $q(\cdot)$ is equal to $2 N /(N-2)$ at a point, then does the problem

$$
-\Delta u(x)=|u(x)|^{q(x)-2} u(x) \quad \text { in } \Omega \quad \text { and } \quad u(x)=0 \quad \text { on } \partial \Omega
$$

have a positive solution? When $q(\cdot)$ is a constant, problem (1.1) has been studied by many researchers. If $q(\cdot)$ is a constant smaller than $2 N /(N-2)$, then the embedding from $W_{0}^{1,2}(\Omega)$ to $L^{q}(\cdot)(\Omega)$ is compact, and hence the existence of a positive solution to $(1.1)$ is easily obtained by the standard mountain pass theorem. When $q(\cdot) \equiv$ $2 N /(N-2)$, problem (1.1) is quite interesting. If $\Omega$ is star-shaped, then Pohozaev [31] showed that there is no solution. If $\Omega$ has a nontrivial topology in the sense of $\mathbf{Z}_{2^{-}}$ homology, then Bahri and Coron [3] showed that the problem has a positive solution; see also [7]. Even if $\Omega$ is contractible, then, under some condition on the shape of $\Omega$, Passaseo [30] obtained a positive solution. In the case when $q(\cdot)$ is a variable exponent and $q(\cdot)$ coincides with $2 N /(N-2)$ at a point in $\Omega$, since the embedding of $W_{0}^{1,2}(\Omega)$ to $L^{q(\cdot)}(\Omega)$ may not be compact, the existence of positive solution to (1.1) is not trivial. Kurata and the fourth author showed that if there exist $x_{0} \in \Omega, C_{0}>0$, $\eta>0$ and $0<l<1$ such that $\operatorname{ess}^{\sup _{x \in \Omega \backslash B_{\eta}\left(x_{0}\right)} q(x)<2 N /(N-2) \text { and }}$

$$
q(x) \leq \frac{2 N}{N-2}-\frac{C_{0}}{\left(\log \left(1 /\left|x-x_{0}\right|\right)\right)^{l}} \quad \text { for almost every } x \in \Omega \cap B_{\eta}\left(x_{0}\right),
$$

then the embedding from $W_{0}^{1,2}(\Omega)$ to $L^{q(\cdot)}(\Omega)$ is compact; see [23, Theorem 2]. As an application of the compact embedding, they obtained a positive solution to (1.1).

Our first aim in this paper is to establish the compact embedding from $W_{0}^{k, p(\cdot)}(\Omega)$ to $L^{q(\cdot)}(\Omega)$ when $q(\cdot)$ is an exponent satisfying a condition weaker than (1.2). As an application, we show the existence of a nontrivial nonnegative weak solution to the nonlinear elliptic equation

$$
\left\{\begin{aligned}
-\operatorname{div}\left(|\nabla u(x)|^{p(x)-2} \nabla u(x)\right) & =|u(x)|^{q(x)-2} u(x) & & \text { in } \Omega, \\
u(x) & =0 & & \text { on } \partial \Omega .
\end{aligned}\right.
$$

Here $u$ is called a weak solution of $(1.3)$ if $u \in W_{0}^{1, p(\cdot)}(\Omega)$ and

$$
\int_{\Omega}\left(|\nabla u(x)|^{p(x)-2} \nabla u(x) \nabla v(x)-|u(x)|^{q(x)-2} u(x) v(x)\right) d x=0
$$

for all $v \in W_{0}^{1, p(\cdot)}(\Omega)$. Our final goal is to find nontrivial nonnegative weak solutions to (1.3), even if the embedding might not be compact.

\section{Preliminaries}

Throughout this paper, we use the symbol $C$ to denote various positive constants independent of the variables in question. We only use $N$ as the dimension of the Euclidean space $\mathbf{R}^{N}$ and we set $B_{r}(x)=\left\{y \in \mathbf{R}^{N}:|y-x|<r\right\}$ for $x \in \mathbf{R}^{N}$ and $r>0$. For a measurable subset $E$ of $\mathbf{R}^{N}$, we denote by $|E|$ the Lebesgue measure of $E$. For a measurable function $u$, we set $u^{+}=\max \{u, 0\}$. Unless otherwise stated, we assume that $N \geq 2$ and $\Omega$ is a bounded open set in $\mathbf{R}^{N}$. 
A measurable function $p(\cdot): \Omega \rightarrow[1, \infty)$ is called a variable exponent on $\Omega$. We set

$$
p_{*}=\underset{x \in \Omega}{\operatorname{essinf}} p(x) \quad \text { and } \quad p^{*}=\operatorname{ess~sup}_{x \in \Omega} p(x) .
$$

It is worth noting the next result, which follows readily from the definition of $L^{p(\cdot)}$-norm (see [17, Theorem 1.3]).

Lemma 2.1. If $p(\cdot)$ is a variable exponent on $\Omega$ satisfying $1 \leq p_{*} \leq p^{*}<\infty$, then

$$
\min \left\{\|u\|_{L^{p(\cdot)}(\Omega)}^{p_{*}},\|u\|_{L^{p(\cdot)}(\Omega)}^{p^{*}}\right\} \leq \int_{\Omega}|u(x)|^{p(x)} d x \leq \max \left\{\|u\|_{L^{p(\cdot)}(\Omega)}^{p_{*}},\|u\|_{L^{p(\cdot)}(\Omega)}^{p^{*}}\right\} .
$$

A variable exponent $p(\cdot)$ is said to satisfy the log-Hölder condition on $\Omega$ if

$$
|p(x)-p(y)| \leq \frac{C}{\log (1 /|x-y|)} \quad \text { for each } x, y \in \Omega \text { with }|x-y|<\frac{1}{2},
$$

where $C$ is a positive constant. We set

$$
p_{k}^{\sharp}(x)= \begin{cases}N p(x) /(N-k p(x)) & \text { if } 1 \leq p(x)<N / k, \\ \infty & \text { if } p(x) \geq N / k\end{cases}
$$

for each $k \in \mathbf{N}$.

We know the following Sobolev inequality for functions in $W_{0}^{1, p(\cdot)}(\Omega)$; see $[20$, Proposition $4.2(1)$ ].

Lemma 2.2. Let $p(\cdot)$ be a variable exponent on $\Omega$ satisfying the log-Hölder condition and $1 \leq p_{*} \leq p^{*}<\infty$. If $p^{*}<N$, then there exists a constant $C>0$ such that

for $u \in W_{0}^{1, p(\cdot)}(\Omega)$.

$$
\|u\|_{L^{p_{1}^{\sharp}(\cdot)}(\Omega)} \leq C\|\nabla u\|_{L^{p(\cdot)}(\Omega)}
$$

Corollary 2.3. Let $p(\cdot)$ be as in the previous lemma. If $p^{*}<N / k$ with $k \in \mathbf{N}$, then there exists a constant $C>0$ such that

$$
\|u\|_{L^{p_{k}^{\sharp}(\cdot)}(\Omega)} \leq C \sum_{|\alpha|=k}\left\|D^{\alpha} u\right\|_{L^{p(\cdot)}(\Omega)}
$$

for $u \in W_{0}^{k, p(\cdot)}(\Omega)$.

Proof. Assume $p^{*}<N / k$ with $k \in \mathbf{N}$. Let $u \in W_{0}^{k, p(\cdot)}(\Omega)$ and let $\ell$ be a positive integer with $\ell \leq k$. Then we see from Lemma 2.2 that $u \in W_{0}^{k-\ell, p_{\ell}^{\sharp}(\cdot)}(\Omega)$, so that

$$
\left\|D^{\alpha} u\right\|_{L^{p_{\ell}^{\sharp}(\cdot)}(\Omega)} \leq C \sum_{|\beta|=k-\ell+1}\left\|D^{\beta} u\right\|_{L^{p_{\ell-1}^{\sharp(\cdot)}(\Omega)}}
$$

for $|\alpha|=k-\ell$, where $p_{0}^{\sharp}(x)=p(x)$. This proves the required result.

\section{Compact embeddings}

In this section, we assume that $p(\cdot)$ is a variable exponent on $\Omega$ satisfying the $\log$-Hölder condition and $1 \leq p_{*} \leq p^{*}<\infty$. For a set $K$ in $\mathbf{R}^{N}$, we define

$$
K(r)=\left\{x \in \mathbf{R}^{N}: \delta_{K}(x) \leq r\right\} \text { for } r>0,
$$

where $\delta_{K}(x)$ denotes the distance of $x$ to $K$. 
First, as in [23], we show the following noncompact embedding from $W_{0}^{k, p(\cdot)}(\Omega)$ to $L^{q(\cdot)}(\Omega)$.

Proposition 3.1. Let $x_{0} \in \Omega$ and $k \in \mathbf{N}$, and let $q(\cdot): \Omega \rightarrow[1, \infty)$ be a variable exponent on $\Omega$ such that there exist $C>0$ and $\eta>0$ satisfying

$$
q(x) \geq p_{k}^{\sharp}(x)-\frac{C}{\log \left(1 /\left|x-x_{0}\right|\right)} \quad \text { for almost every } x \in \Omega \cap B_{\eta}\left(x_{0}\right) .
$$

If $p\left(x_{0}\right)<N / k$, then the embedding from $W_{0}^{k, p(\cdot)}(\Omega)$ to $L^{q(\cdot)}(\Omega)$ is not compact.

Proof. Assume $p\left(x_{0}\right)<N / k$. We may assume that $x_{0}=0$ and $B_{1}(0) \subset \Omega$. Let $\psi \in C_{0}^{\infty}(\mathbf{R})$ be a function such that $0 \leq \psi(r) \leq 1, \psi(r)=0$ for $r>1$ and $\psi(r)=1$ for $0 \leq r<1 / 2$. Set

$$
\psi_{n}(x)=n^{N / p_{k}^{\sharp}(0)} \psi(n|x|)
$$

for each $n \in \mathbf{N}$. Then, for $n \geq 2$ and $0 \leq|\alpha| \leq k$, we note

$$
\begin{aligned}
\int_{\Omega}\left|D^{\alpha} \psi_{n}(x)\right|^{p(x)} d x & \leq C \int_{B_{1 / n}(0)} n^{\left(N / p_{k}^{\sharp}(0)+|\alpha|\right) p(x)} d x \\
& \leq C n^{\left(N / p_{k}^{\sharp}(0)+|\alpha|\right)(p(0)+C / \log n)} \int_{B_{1 / n}(0)} d x \leq C
\end{aligned}
$$

by the log-Hölder condition on $p(\cdot)$. Using (3.1), we have

$$
\int_{\Omega}\left|\psi_{n}(x)\right|^{q(x)} d x \geq \int_{B_{1 /(2 n)}(0)} n^{N q(x) / p_{k}^{\sharp}(0)}|\psi(n|x|)|^{q(x)} d x \geq C n^{N} \int_{B_{1 /(2 n)}(0)} d x=C>0,
$$

which implies that the embedding from $W_{0}^{k, p(\cdot)}(\Omega)$ to $L^{q(\cdot)}(\Omega)$ is not compact since $\int_{\Omega}\left|\psi_{n}(x)\right|^{p(x)} d x \rightarrow 0$ as $n \rightarrow \infty$.

As a direct consequence, we have the following result:

Corollary 3.2. Let $K$ be a set in $\mathbf{R}^{N}$, and let $x_{0} \in K \cap \Omega$ and $k \in \mathbf{N}$. Let $q(\cdot): \Omega \rightarrow[1, \infty)$ be a variable exponent on $\Omega$ such that there exist $C>0$ and $r>0$ satisfying

$$
q(x) \geq p_{k}^{\sharp}(x)-\frac{C}{\log \left(1 / \delta_{K}(x)\right)} \quad \text { for almost every } x \in K(r) \cap \Omega .
$$

If $p\left(x_{0}\right)<N / k$, then the embedding from $W_{0}^{k, p(\cdot)}(\Omega)$ to $L^{q(\cdot)}(\Omega)$ is not compact.

Proof. Assume $p\left(x_{0}\right)<N / k$. Since $\delta_{K}(x) \leq\left|x-x_{0}\right|$ for each $x \in \mathbf{R}^{N}$, we obtain the conclusion by the previous proposition.

For the compact embeddings, we first give the following result.

Proposition 3.3. Assume that $p^{*}<N / k$ with some $k \in \mathbf{N}$. Let $q(\cdot)$ be a variable exponent on $\Omega$ such that $1 \leq q_{*}$ and

$$
\underset{x \in \Omega}{\operatorname{essinf}}\left(p_{k}^{\sharp}(x)-q(x)\right)>0 \text {. }
$$

Then the following hold.

(i) The embedding of $W_{0}^{k, p(\cdot)}(\Omega)$ to $L^{q(\cdot)}(\Omega)$ is compact.

(ii) If $\Omega$ satisfies the cone condition, then the embedding of $W^{k, p(\cdot)}(\Omega)$ to $L^{q(\cdot)}(\Omega)$ is compact. 
The case (i) in the proposition is essentially a special case of [22, Theorem 3.8]; the case (ii) is a slight generalization of [14, Theorem 1.3] to the case $1 \leq p_{*}$.

Proof of Proposition 3.3. We only give a proof of (ii), since (i) can be proved similarly. Assume that $\Omega$ satisfies the cone condition. By (3.2), take $\varepsilon>0$ such that $p_{k}^{\sharp}(x)-q(x)>2 \varepsilon>0$ for almost every $x \in \Omega$. Since $p(\cdot)$ is uniformly continuous on $\Omega$, one can find open balls $\left\{B_{j}\right\}_{j=1}^{l}$ and $\left\{\tilde{B}_{j}\right\}_{j=1}^{l}$ with $l \in \mathbf{N}$ such that $\bar{\Omega} \subset \bigcup_{i=1}^{l} B_{i}$, $\bar{B}_{j} \subset \tilde{B}_{j}$ and

$$
\inf _{x \in \tilde{B}_{j} \cap \Omega} p_{k}^{\sharp}(x)-\varepsilon \geq \sup _{x \in \tilde{B}_{j} \cap \Omega} p_{k}^{\sharp}(x)-2 \varepsilon \geq \operatorname{ess}_{x \in \tilde{B}_{j} \cap \Omega} q(x) \quad \text { for each } j=1, \ldots, l .
$$

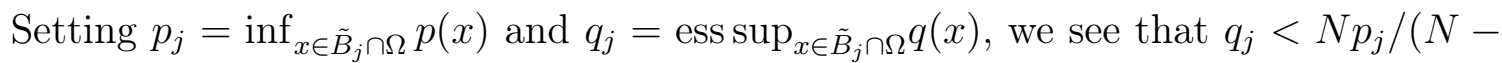
$\left.k p_{j}\right)$ and the embedding from $\left\{u \in W^{k, p(\cdot)}(\Omega): u=0\right.$ on $\left.\Omega \backslash \tilde{B}_{j}\right\}$ to $W^{k, p_{j}}(\Omega)$ and the embedding from $\left\{u \in L^{q_{j}}(\Omega): u=0\right.$ on $\left.\Omega \backslash \tilde{B}_{j}\right\}$ to $L^{q(\cdot)}(\Omega)$ are continuous. By the Rellich-Kondrachov theorem (see [1, Theorem 6.3]), $W^{k, p_{j}}(\Omega)$ is compactly embedded into $L^{q_{j}}(\Omega)$. Now, take $\varphi_{j} \in C^{1}(\Omega ;[0,1])$ such that $\left|\nabla \varphi_{j}\right| \leq C$ on $\Omega, \varphi_{j}=1$ on $\Omega \cap B_{j}$ and $\varphi_{j}=0$ on $\Omega \backslash \tilde{B}_{j}$. It is easy to see that the linear operator $u \mapsto \varphi_{j} u$ is continuous on $W^{k, p(\cdot)}(\Omega)$. Noting $\varphi_{j} u=0$ on $\Omega \backslash \tilde{B}_{j}$ for each $u \in W^{k, p(\cdot)}(\Omega)$, we can infer that $\left\{\varphi_{j} u: u \in W^{k, p(\cdot)}(\Omega)\right\}$ is compactly embedded into $L^{q(\cdot)}(\Omega)$. Passing to subsequences repeatedly, we obtain the conclusion.

For a compact set $K$ in $\mathbf{R}^{N}$ and $s \in[0, N]$, following Mattila [25], we say that the $(N-s)$-dimensional upper Minkowski content of $K$ is finite if

$$
|K(r)| \leq C r^{s} \quad \text { for small } r>0 \text {. }
$$

Now we are concerned with the compact embedding from $W_{0}^{k, p(\cdot)}(\Omega)$ to $L^{q(\cdot)}(\Omega)$ when $q(\cdot)$ and $p_{k}^{\sharp}(\cdot)$ coincides on some part of $\Omega$.

Theorem 3.4. Let $\varphi(\cdot):\left[1 / r_{0}, \infty\right) \rightarrow(0, \infty)$ be a continuous function such that

(i) $\varphi(r) / \log r$ is nonincreasing on $\left[1 / r_{0}, \infty\right)$,

(ii) $\varphi(r) \rightarrow \infty$ as $r \rightarrow \infty$

for some $r_{0} \in(0,1 / e)$. Let $K$ be a compact set in $\mathbf{R}^{N}$ whose $(N-s)$-dimensional upper Minkowski content is finite for some $s$ with $0<s \leq N$. Let $k \in \mathbf{N}$ and let $q(\cdot)$ be a variable exponent on $\Omega$ such that

(iii) $1 \leq q_{*} \leq q^{*}<\infty$,

(iv) $\operatorname{essinf}_{\Omega \backslash K\left(r_{0}\right)}\left(p_{k}^{\sharp}(x)-q(x)\right)>0$,

(v) $q(x) \leq p_{k}^{\sharp}(x)-\frac{\varphi\left(1 / \delta_{K}(x)\right)}{\log \left(1 / \delta_{K}(x)\right)}$ for almost every $x \in K\left(r_{0}\right) \cap \Omega$.

Then the embedding from $W_{0}^{k, p(\cdot)}(\Omega)$ to $L^{q(\cdot)}(\Omega)$ is compact.

Proof. Without loss of generality, we may assume $\varphi(r) / \log r \rightarrow 0$ as $r \rightarrow \infty$; otherwise, we have ess $\inf _{x \in \Omega}\left(p_{k}^{\sharp}(x)-q(x)\right)>0$, so that the conclusion follows from Proposition 3.3 (i).

First, consider the case $p^{*}<N / k$. Let us prove that

$$
\lim _{\varepsilon \rightarrow+0} \sup \left\{\int_{K(\varepsilon) \cap \Omega}|v(x)|^{q(x)} d x: v \in W_{0}^{k, p(\cdot)}(\Omega),\|v\|_{W^{k, p(\cdot)}(\Omega)} \leq 1\right\}=0 .
$$


For this purpose, take $\beta$ with $0<\beta<s /\left(p^{*}\right)_{k}^{\sharp}$. Let $\varepsilon>0$ such that $\varepsilon^{-1}>1 / r_{0}$ and $\varphi(1 / \varepsilon) \geq 1$. We set $\eta_{n}=\varepsilon^{-\beta n}$ for each $n \in \mathbf{N}$. Then, by the assumptions on $\varphi$, we have for each $n \in \mathbf{N}$ and $x \in\left(K\left(\varepsilon^{n}\right) \backslash K\left(\varepsilon^{n+1}\right)\right) \cap \Omega$,

$$
\eta_{n}^{q(x)-p_{k}^{\sharp}(x)} \leq \eta_{n}^{-\frac{\varphi\left(1 / \delta_{K}(x)\right)}{\log \left(1 / \delta_{K}(x)\right)}} \leq \eta_{n}^{-\frac{\varphi\left(1 / \varepsilon^{n+1}\right)}{\log \left(1 / \varepsilon^{n+1}\right)}}=\exp \left(-(\beta n /(n+1)) \varphi\left(1 / \varepsilon^{n+1}\right)\right) \equiv A_{n} .
$$

Since

$$
|K(r) \cap \Omega| \leq C r^{s} \quad \text { for all } r>0
$$

by the boundedness of $\Omega$, we have

$$
\int_{\left(K\left(\varepsilon^{n}\right) \backslash K\left(\varepsilon^{n+1}\right)\right) \cap \Omega} \eta_{n}^{q(x)} d x \leq \eta_{n}^{\left(p^{*}\right)_{k}^{\sharp}} \int_{K\left(\varepsilon^{n}\right) \cap \Omega} d x \leq C \varepsilon^{n\left(s-\beta\left(p^{*}\right)_{k}^{\sharp}\right)} .
$$

Hence we have

$$
\begin{aligned}
& \int_{\left(K\left(\varepsilon^{n}\right) \backslash K\left(\varepsilon^{n+1}\right)\right) \cap \Omega}|v(x)|^{q(x)} d x \\
& \leq \int_{\left(K\left(\varepsilon^{n}\right) \backslash K\left(\varepsilon^{n+1}\right)\right) \cap \Omega}|v(x)|^{q(x)}\left(\frac{|v(x)|}{\eta_{n}}\right)^{p_{k}^{\sharp}(x)-q(x)} d x+\int_{\left(K\left(\varepsilon^{n}\right) \backslash K\left(\varepsilon^{n+1}\right)\right) \cap \Omega} \eta_{n}^{q(x)} d x \\
& \leq A_{n} \int_{\left(K\left(\varepsilon^{n}\right) \backslash K\left(\varepsilon^{n+1}\right)\right) \cap \Omega}|v(x)|^{p_{k}^{\sharp}(x)} d x+C \varepsilon^{n\left(s-\beta\left(p^{*}\right)_{k}^{\sharp}\right)},
\end{aligned}
$$

so that for each $n_{0} \in \mathbf{N}$, we obtain

$$
\begin{aligned}
\int_{K\left(\varepsilon^{n_{0}}\right) \cap \Omega}|v(x)|^{q(x)} d x & =\sum_{n=n_{0}}^{\infty} \int_{\left(K\left(\varepsilon^{n}\right) \backslash K\left(\varepsilon^{n+1}\right)\right) \cap \Omega}|v(x)|^{q(x)} d x \\
& \leq\left(\sup _{n \geq n_{0}} A_{n}\right) \int_{\Omega}|v(x)|^{p_{k}^{\sharp}(x)} d x+C \sum_{n=n_{0}}^{\infty} \varepsilon^{n\left(s-\beta\left(p^{*}\right)_{k}^{\sharp}\right)} .
\end{aligned}
$$

Since $A_{n} \rightarrow 0$ as $n \rightarrow \infty, s-\beta\left(p^{*}\right)_{k}^{\sharp}>0$ and $\|v\|_{L_{k}^{p_{k}^{\sharp(\cdot)}(\Omega)}} \leq C\|v\|_{W^{k, p(\cdot)}(\Omega)}$ for all $v \in W_{0}^{k, p(\cdot)}(\Omega)$ by Corollary 2.3, (3.3) is obtained by letting $n_{0} \rightarrow \infty$.

Let $\left\{v_{j}\right\}$ be a bounded sequence in $W_{0}^{k, p(\cdot)}(\Omega)$. We may assume that it converges weakly to some $v \in W_{0}^{k, p(\cdot)}(\Omega)$. By Proposition 3.3 (ii), the embedding from $W^{k, p(\cdot)}(B)$ to $L^{q(\cdot)}(B)$ is compact for each ball $B \subset \Omega$ such that $\operatorname{ess}_{\inf } \operatorname{seB}_{x \in B}\left(p_{k}^{\sharp}(x)-\right.$ $q(x))>0$. Let $n \in \mathbf{N}$. Since $\Omega \backslash K\left(2^{-n}\right)$ is a bounded open set in $\mathbf{R}^{N}$, there exists a finite family of balls contained in $\mathbf{R}^{N} \backslash K\left(2^{-n-1}\right)$ whose union contains $\Omega \backslash K\left(2^{-n}\right)$. Since ess $\inf _{x \in \Omega \backslash K\left(2^{-n-1}\right)}\left(p_{k}^{\sharp}(x)-q(x)\right)>0$, we can find a subsequence $\left\{v_{j_{k}, n}\right\}$ of $\left\{v_{j}\right\}$ such that $v_{j_{k}, n} \rightarrow v$ in $L^{q(\cdot)}\left(\Omega \backslash K\left(2^{-n}\right)\right)$ as well as almost everywhere on $\Omega \backslash K\left(2^{-n}\right)$. Using the diagonal method, we can find a subsequence $\left\{v_{j_{n}}\right\}$ such that $v_{j_{n}} \rightarrow v$ in $L^{q(\cdot)}(\Omega \backslash K(\varepsilon))$ for each small $\varepsilon>0$ and $v_{j_{n}} \rightarrow v$ almost everywhere on $\Omega$. It follows that

$$
\begin{aligned}
& \varlimsup_{n \rightarrow \infty} \int_{\Omega}\left|v_{j_{n}}(x)-v(x)\right|^{q(x)} d x \\
& =\varlimsup_{n \rightarrow \infty}\left(\int_{K(\varepsilon) \cap \Omega}\left|v_{j_{n}}(x)-v(x)\right|^{q(x)} d x+\int_{\Omega \backslash K(\varepsilon)}\left|v_{j_{n}}(x)-v(x)\right|^{q(x)} d x\right) \\
& =\varlimsup_{n \rightarrow \infty} \int_{K(\varepsilon) \cap \Omega}\left|v_{j_{n}}(x)-v(x)\right|^{q(x)} d x
\end{aligned}
$$


for each small $\varepsilon>0$, which together with (3.3) implies that $\left\|v_{j_{n}}-v\right\|_{L^{q(\cdot)}(\Omega)} \rightarrow 0$ as $n \rightarrow \infty$.

Next consider the general case. We choose $\varepsilon_{0}>0$ such that

$$
q^{*}<N\left(N / k-\varepsilon_{0}\right) /\left(k \varepsilon_{0}\right)-\varphi\left(1 / r_{0}\right) / \log \left(1 / r_{0}\right) .
$$

We set $p_{\varepsilon_{0}}(x)=\min \left\{p(x), N / k-\varepsilon_{0}\right\}$. Since the embedding from $W_{0}^{k, p(\cdot)}(\Omega)$ to $W_{0}^{k, p_{\varepsilon_{0}}(\cdot)}(\Omega)$ is bounded, we can apply the first considerations to obtain the required result.

As a special case of Theorem 3.4, we have the following corollary, which gives an extension of [23, Theorem 2]. We put $\log ^{1} r=\log r$ and $\log ^{n+1} r=\log \left(\log ^{n} r\right)$, inductively.

Corollary 3.5. Let $k \in \mathbf{N}$ and let $q(\cdot)$ be a variable exponent on $\Omega$ such that $1 \leq q_{*} \leq q^{*}<\infty$. Suppose there exist $x_{0} \in \Omega, C>0, n \in \mathbf{N}$ and small $r_{0}>0$ such that

$$
\underset{x \in \Omega \backslash B_{r_{0}}\left(x_{0}\right)}{\operatorname{essinf}}\left(p_{k}^{\sharp}(x)-q(x)\right)>0
$$

and

$$
q(x) \leq p_{k}^{\sharp}(x)-\frac{C \log ^{n}\left(1 /\left|x-x_{0}\right|\right)}{\log \left(1 /\left|x-x_{0}\right|\right)} \quad \text { for almost every } x \in B_{r_{0}}\left(x_{0}\right) .
$$

Then the embedding from $W_{0}^{k, p(\cdot)}(\Omega)$ to $L^{q(\cdot)}(\Omega)$ is compact.

\section{Existence of a solution to (1.3): compact embedding case}

In this section, we assume that $p(\cdot)$ is a variable exponent on $\Omega$ satisfying the log-Hölder condition and $1<p_{*} \leq p^{*}<N$. Further let $q(\cdot)$ be a variable exponent on $\Omega$ such that $p^{*}<q_{*} \leq q(x) \leq p_{1}^{\sharp}(x)$ for almost every $x \in \Omega$.

As an application of Theorem 3.4, we show an existence result of nontrivial nonnegative weak solutions to (1.3) as follows.

Theorem 4.1. Assume that the embedding from $W_{0}^{1, p(\cdot)}(\Omega)$ to $L^{q(\cdot)}(\Omega)$ is compact. Then there exists a nontrivial nonnegative weak solution of (1.3).

In the case of ess $\inf _{x \in \Omega}\left(p_{1}^{\sharp}(x)-q(x)\right)>0$, Fan and Zhang obtained such a result in [15, Theorem 4.7]. Although $q(\cdot)$ can be equal to $p_{1}^{\sharp}(\cdot)$ at some points, the proof in [15] also works in our case with minor changes since we consider the case that the embedding from $W_{0}^{1, p(\cdot)}(\Omega)$ to $L^{q(\cdot)}(\Omega)$ is compact. However, for the reader's convenience, we give a proof of our theorem.

Let $X$ be a Banach space. We say that $u \in X$ is a critical point of $I \in C^{1}(X ; \mathbf{R})$ if the Fréchet derivative $I^{\prime}(u)$ of $I$ at $u$ is zero. We say that $\left\{u_{n}\right\} \subset X$ is a Palais-Smale sequence for $I$ if $\left\{I\left(u_{n}\right)\right\}$ is bounded and $I^{\prime}\left(u_{n}\right) \rightarrow 0$ as $n \rightarrow \infty$ in the dual space of $X$. We also say that $I$ satisfies the Palais-Smale condition if every Palais-Smale sequence for $I$ has a convergent subsequence.

We consider a functional $I: W_{0}^{1, p(\cdot)}(\Omega) \rightarrow \mathbf{R}$ defined by

$$
I(u)=\int_{\Omega}\left(\frac{1}{p(x)}|\nabla u(x)|^{p(x)}-\frac{1}{q(x)} u^{+}(x)^{q(x)}\right) d x \quad \text { for } u \in W_{0}^{1, p(\cdot)}(\Omega) .
$$


The Gâteaux derivative $I^{\prime}(u)$ of $I$ at $u \in W_{0}^{1, p(\cdot)}(\Omega)$ is given by

$$
\begin{aligned}
\left\langle I^{\prime}(u), v\right\rangle & =\lim _{t \rightarrow 0} \frac{I(u+t v)-I(u)}{t} \\
& =\int_{\Omega}\left(|\nabla u(x)|^{p(x)-2} \nabla u(x) \nabla v(x)-u^{+}(x)^{q(x)-1} v(x)\right) d x
\end{aligned}
$$

for each $v \in W_{0}^{1, p(\cdot)}(\Omega)$. By the Vitali convergence theorem, we see that $I^{\prime}$ is continuous from $W_{0}^{1, p(\cdot)}(\Omega)$ to its dual space $\left(W_{0}^{1, p(\cdot)}(\Omega)\right)^{\prime}$, and hence $I \in C^{1}\left(W_{0}^{1, p(\cdot)}(\Omega) ; \mathbf{R}\right)$.

The following is essentially due to Boccardo and Murat [4, Theorem 2.1].

Proposition 4.2. Let $\left\{u_{n}\right\} \subset W_{0}^{1, p(\cdot)}(\Omega)$ be a Palais-Smale sequence for $I$. Then $\left\{u_{n}\right\}$ is bounded in $W_{0}^{1, p(\cdot)}(\Omega)$. Further there exist a subsequence $\left\{u_{n_{i}}\right\}$ of $\left\{u_{n}\right\}$ and $u \in W_{0}^{1, p(\cdot)}(\Omega)$ such that $\left\{\nabla u_{n_{i}}(x)\right\}$ converges to $\nabla u(x)$ for almost every $x \in \Omega$.

Proof. Setting $\beta=\sup _{n \in \mathbf{N}} I\left(u_{n}\right)$, we have

$$
\int_{\Omega}\left(\frac{1}{p^{*}}\left|\nabla u_{n}(x)\right|^{p(x)}-\frac{1}{q_{*}} u_{n}^{+}(x)^{q(x)}\right) d x \leq I\left(u_{n}\right) \leq \beta \quad \text { for all } n \in \mathbf{N} .
$$

Since $I^{\prime}\left(u_{n}\right) \rightarrow 0$ as $n \rightarrow \infty$ in $\left(W_{0}^{1, p(\cdot)}(\Omega)\right)^{\prime}$, we have

$$
\int_{\Omega}\left(\left|\nabla u_{n}(x)\right|^{p(x)}-u_{n}^{+}(x)^{q(x)}\right) d x=\left\langle I^{\prime}\left(u_{n}\right), u_{n}\right\rangle \geq-\left\|u_{n}\right\|_{W^{1, p(\cdot)}(\Omega)}
$$

for each large positive integer $n$. Subtracting (4.2) divided by $q_{*}$ from (4.1) gives

$$
\left(\frac{1}{p^{*}}-\frac{1}{q_{*}}\right) \int_{\Omega}\left|\nabla u_{n}(x)\right|^{p(x)} d x \leq \beta+\frac{1}{q_{*}}\left\|u_{n}\right\|_{W^{1, p(\cdot)}(\Omega)} \leq C\left(\left\|\nabla u_{n}\right\|_{L^{p(\cdot)(\Omega)}}+1\right) ;
$$

we used Lemma 2.2 in the second inequality. Thus Lemma 2.1 gives

$$
\left\|\nabla u_{n}\right\|_{L^{p(\cdot)(\Omega)}}+1 \geq C \min \left\{\left\|\nabla u_{n}\right\|_{L^{p(\cdot)}(\Omega)}^{p_{*}},\left\|\nabla u_{n}\right\|_{L^{p(\cdot)}(\Omega)}^{p^{*}}\right\}
$$

so that $\left\{u_{n}\right\}$ is bounded in $W_{0}^{1, p(\cdot)}(\Omega)$. Hence, passing to a subsequence, we may assume that $\left\{u_{n}\right\}$ converges weakly to some $u$ in $W_{0}^{1, p(\cdot)}(\Omega)$ and $\left\{u_{n}(x)\right\}$ converges to $u(x)$ for almost every $x \in \Omega$. For $\eta>0$, let $T_{\eta}: \mathbf{R} \rightarrow \mathbf{R}$ be a function such that

$$
T_{\eta}(t)=t \quad \text { for }|t| \leq \eta, \quad T_{\eta}(t)=\eta t /|t| \quad \text { for }|t|>\eta .
$$

Since $\left\{T_{\eta}\left(u_{n}-u\right)\right\}$ converges weakly to 0 in $W_{0}^{1, p(\cdot)}(\Omega)$ and $\left\{u_{n}\right\}$ is bounded in $L^{q(\cdot)}(\Omega)$ by Lemma 2.2 , we have

$$
\begin{aligned}
& \varlimsup_{n \rightarrow \infty} \int_{\Omega}\left(\left|\nabla u_{n}(x)\right|^{p(x)-2} \nabla u_{n}(x)-|\nabla u(x)|^{p(x)-2} \nabla u(x)\right) \nabla\left(T_{\eta}\left(u_{n}(x)-u(x)\right)\right) d x \\
& =\varlimsup_{n \rightarrow \infty} \int_{\Omega} u_{n}^{+}(x)^{q(x)-1} T_{\eta}\left(u_{n}(x)-u(x)\right) d x \leq C \eta,
\end{aligned}
$$

where $C>0$ is a constant which is independent of $\eta>0$. We set

$$
\rho_{n}(x)=\left(\left|\nabla u_{n}(x)\right|^{p(x)-2} \nabla u_{n}(x)-|\nabla u(x)|^{p(x)-2} \nabla u(x)\right)\left(\nabla u_{n}(x)-\nabla u(x)\right) .
$$

We note that $\rho_{n} \geq 0$ almost everywhere for each $n \in \mathbf{N}$. Further we set

$$
E_{n}=\left\{x \in \Omega:\left|u_{n}(x)-u(x)\right| \leq \eta\right\}, \quad F_{n}=\left\{x \in \Omega:\left|u_{n}(x)-u(x)\right|>\eta\right\}
$$


for each $n \in \mathbf{N}$. We fix $\theta \in(0,1)$. Since

$\int_{\Omega} \rho_{n}(x)^{\theta} d x \leq\left(\int_{E_{n}} \rho_{n}(x) d x\right)^{\theta}\left|E_{n}\right|^{1-\theta}+\left(\int_{F_{n}} \rho_{n}(x) d x\right)^{\theta}\left|F_{n}\right|^{1-\theta} \quad$ for each $n \in \mathbf{N}$, $\left|F_{n}\right| \rightarrow 0$ and $\left\{\rho_{n}\right\}$ is bounded in $L^{1}(\Omega)$, we have

$$
\varlimsup_{n \rightarrow \infty} \int_{\Omega} \rho_{n}(x)^{\theta} d x \leq(C \eta)^{\theta}|\Omega|^{1-\theta} .
$$

Letting $\eta \rightarrow 0$, we have $\int_{\Omega} \rho_{n}(x)^{\theta} d x \rightarrow 0$. Thus we may assume $\left\{\rho_{n}(x)\right\}$ converges to 0 for almost every $x \in \Omega$. Since $p_{*}>1$, we see that a subsequence of $\left\{\nabla u_{n}(x)\right\}$ converges to $\nabla u(x)$ for almost every $x \in \Omega$.

Lemma 4.3. Suppose the embedding from $W_{0}^{1, p(\cdot)}(\Omega)$ to $L^{q(\cdot)}(\Omega)$ is compact. Then the functional I satisfies the Palais-Smale condition.

Proof. Let $\left\{u_{n}\right\} \subset W_{0}^{1, p(\cdot)}(\Omega)$ be a Palais-Smale sequence for $I$. By the previous proposition, we may assume that $\left\{u_{n}\right\}$ converges weakly to some $u \in W_{0}^{1, p(\cdot)}(\Omega)$, and $\left\{u_{n}(x)\right\}$ and $\left\{\nabla u_{n}(x)\right\}$ converge to $u(x)$ and $\nabla u(x)$ almost every $x \in \Omega$, respectively. Since $\left\langle I^{\prime}\left(u_{n}\right), u\right\rangle \rightarrow 0$, the Vitali convergence theorem implies that

$$
\int_{\Omega}|\nabla u(x)|^{p(x)} d x=\int_{\Omega} u^{+}(x)^{q(x)} d x .
$$

This equality together with $\left\langle I^{\prime}\left(u_{n}\right), u_{n}\right\rangle \rightarrow 0$ and the compact embedding assumption give

$$
\begin{aligned}
\lim _{n \rightarrow \infty} \int_{\Omega}\left|\nabla u_{n}(x)\right|^{p(x)} d x & =\lim _{n \rightarrow \infty} \int_{\Omega} u_{n}^{+}(x)^{q(x)} d x \\
& =\int_{\Omega} u^{+}(x)^{q(x)} d x=\int_{\Omega}|\nabla u(x)|^{p(x)} d x .
\end{aligned}
$$

Now, we consider the function

$$
w_{n}(x)=2^{p^{*}-1}\left(\left|\nabla u_{n}(x)\right|^{p(x)}+|\nabla u(x)|^{p(x)}\right)-\left|\nabla u_{n}(x)-\nabla u(x)\right|^{p(x)} .
$$

Since $w_{n}(x) \geq 0$ for almost every $x \in \Omega$, we see from Fatou's lemma and (4.3) that

$$
\begin{aligned}
& 2^{p^{*}} \int_{\Omega}|\nabla u(x)|^{p(x)} d x-\varlimsup_{n \rightarrow \infty} \int_{\Omega}\left|\nabla u_{n}(x)-\nabla u(x)\right|^{p(x)} d x \\
& \geq \int_{\Omega} \underline{\lim } w_{n}(x) d x=2^{p^{*}} \int_{\Omega}|\nabla u(x)|^{p(x)} d x,
\end{aligned}
$$

so that

$$
\lim _{n \rightarrow \infty} \int_{\Omega}\left|\nabla u_{n}(x)-\nabla u(x)\right|^{p(x)} d x=0 .
$$

Hence we see that $\left\{u_{n}\right\}$ converges strongly to $u$ in $W_{0}^{1, p(\cdot)}(\Omega)$.

We recall the following variant of the mountain pass theorem; see e.g., [34].

Theorem 4.4. Let $X$ be a Banach space and let $I$ be a $C^{1}$ functional on $X$ such that $I(0)=0$,

(i) there exist positive constants $\kappa, r>0$ such that $I(u) \geq \kappa$ for all $u \in X$ with $\|u\|=r$, and

(ii) there exists an element $v \in X$ such that $I(v)<0$ and $\|v\|>r$. 
Define

$$
c=\inf _{\gamma \in \Gamma} \max _{0 \leq t \leq 1} I(\gamma(t))
$$

where

$$
\Gamma=\{\gamma \in C([0,1] ; X): \gamma(0)=0, I(\gamma(1))<0,\|\gamma(1)\|>r\} .
$$

Then $c>0$ and for each $\varepsilon>0$, there exists $u \in X$ such that $|I(u)-c| \leq \varepsilon$ and $\left\|I^{\prime}(u)\right\| \leq \varepsilon$.

Now we are ready to prove Theorem 4.1.

Proof of Theorem 4.1.. First we find $r>0$ such that

$$
\inf \left\{I(u): u \in W_{0}^{1, p(\cdot)}(\Omega),\|u\|_{W^{1, p(\cdot)}(\Omega)}=r\right\}>0 .
$$

Taking $r>0$ so small, by Lemma 2.2 , we have $\|\nabla u\|_{L^{p(\cdot)}(\Omega)} \leq 1$ and $\|u\|_{L^{q(\cdot)}(\Omega)} \leq 1$ for all $u \in W_{0}^{1, p(\cdot)}(\Omega)$ with $\|u\|_{W^{1, p(\cdot)}(\Omega)}=r$. Then for each $u \in W_{0}^{1, p(\cdot)}(\Omega)$ with $\|u\|_{W^{1, p(\cdot)(\Omega)}}=r$, we have

$$
\int_{\Omega} u^{+}(x)^{q(x)} d x \leq\|u\|_{L^{q(\cdot)}(\Omega)}^{q_{*}} \leq C\|u\|_{L^{p_{1}^{\sharp}(\cdot)}(\Omega)}^{q_{*}} \leq C\|\nabla u\|_{L^{p(\cdot)}(\Omega)}^{q_{*}}
$$

by Lemmas 2.1 and 2.2, so that

$$
I(u) \geq \frac{1}{p^{*}}\|\nabla u\|_{L^{p(\cdot)}(\Omega)}^{p^{*}}-\frac{C}{q_{*}}\|\nabla u\|_{L^{p(\cdot)}(\Omega)}^{q_{*}}
$$

Since $p^{*}<q_{*}$, we have (4.5) if $r>0$ is small.

Next we prove $I(t u) \rightarrow-\infty$ as $t \rightarrow \infty$ for $u \in W_{0}^{1, p(\cdot)}(\Omega)$ with $u^{+} \neq 0$. In fact, if $u \in W_{0}^{1, p(\cdot)}(\Omega)$ such that $u^{+} \neq 0$, then we see that

$$
I(t u) \leq t^{p^{*}} \int_{\Omega} \frac{1}{p(x)}|\nabla u(x)|^{p(x)} d x-t^{q_{*}} \int_{\Omega} \frac{1}{q(x)} u^{+}(x)^{q(x)} d x \rightarrow-\infty
$$

as $t \rightarrow \infty$, since $p^{*}<q_{*}$.

Now the required result follows from Lemma 4.3 and Theorem 4.4.

As a direct consequence of Theorem 4.1, we have the following:

Corollary 4.5. Suppose all hypotheses in Theorem 3.4 hold for $k=1$. Then there exists a nontrivial nonnegative weak solution of (1.3).

\section{Existence of a solution to (1.3): noncompact embedding case}

Our final aim is to deal with the existence result of a nontrivial nonnegative weak solution to (1.3) in the case that the embedding may not be compact.

For real sequences $\left\{a_{n}\right\}$ and $\left\{b_{n}\right\}$, we write $a_{n}=b_{n}+o(1)$ or $a_{n} \leq b_{n}+o(1)$ if $\lim _{n}\left(a_{n}-b_{n}\right)=0$ or $\varlimsup_{n}\left(a_{n}-b_{n}\right) \leq 0$, respectively.

Proposition 5.1. Let $p(\cdot)$ be a log-Hölder continuous function on $\Omega$ with $1<$ $p_{*} \leq p^{*}<N$ and let $q(\cdot)$ be a measurable function on $\Omega$ such that $p^{*}<q_{*} \leq q(x) \leq$ 
$p_{1}^{\sharp}(x)$ for almost every $x \in \Omega$. Assume $\inf _{u \in \mathscr{N}_{I}} I(u)<\inf _{u \in \mathscr{N}_{J}} J(u)$, where

$$
\begin{aligned}
I(u) & =\int_{\Omega}\left(\frac{1}{p(x)}|\nabla u(x)|^{p(x)}-\frac{1}{q(x)} u^{+}(x)^{q(x)}\right) d x \quad \text { for } u \in W_{0}^{1, p(\cdot)}(\Omega), \\
J(u) & =\int_{\Omega}\left(\frac{1}{p(x)}|\nabla u(x)|^{p(x)}-\frac{1}{p_{1}^{\sharp}(x)} u^{+}(x)^{p_{1}^{\sharp}(x)}\right) d x \quad \text { for } u \in W_{0}^{1, p(\cdot)}(\Omega), \\
\mathscr{N}_{I} & =\left\{u \in W_{0}^{1, p(\cdot)}(\Omega) \backslash\{0\}: \int_{\Omega}|\nabla u(x)|^{p(x)} d x=\int_{\Omega} u^{+}(x)^{q(x)} d x\right\}, \\
\mathscr{N}_{J} & =\left\{u \in W_{0}^{1, p(\cdot)}(\Omega) \backslash\{0\}: \int_{\Omega}|\nabla u(x)|^{p(x)} d x=\int_{\Omega} u^{+}(x)^{p_{1}^{\sharp}(x)} d x\right\} .
\end{aligned}
$$

Then problem (1.3) has a nontrivial nonnegative weak solution.

Proof. We set $c=\inf _{u \in \mathscr{N}_{I}} I(u)$, and define $\Gamma$ by (4.4) with $X=W_{0}^{1, p(\cdot)}(\Omega)$. Along the similar lines as those in the proof of Theorem 4.1, we can easily see that $\Gamma \neq \emptyset$, $\mathscr{N}_{J} \neq \emptyset, \mathscr{N}_{I} \neq \emptyset$ and (4.5) holds for small $r>0$.

First we show

$$
c=\inf _{\gamma \in \Gamma} \max _{0 \leq t \leq 1} I(\gamma(t)) .
$$

Let $u \in \mathscr{N}_{I}$. For $\alpha_{u}>1$ large enough, consider the path $\gamma_{u} \in \Gamma$ defined by $\gamma_{u}(t)=$ $t \alpha_{u} u$ for $t \in[0,1]$. Since $I(u)=\max _{0 \leq t \leq 1} I\left(\gamma_{u}(t)\right)$, we have

$$
c \geq \inf _{\gamma \in \Gamma} \max _{0 \leq t \leq 1} I(\gamma(t)) .
$$

On the other hand, let $\gamma \in \Gamma$. Then

$$
\int_{\Omega}\left(|\nabla \gamma(1)|^{p(x)}-\left(\gamma(1)^{+}\right)^{q(x)}\right) d x<0 .
$$

As in the proof of Theorem 4.1, we find a small $t>0$ satisfying

$$
\int_{\Omega}\left(|\nabla \gamma(t)|^{p(x)}-\left(\gamma(t)^{+}\right)^{q(x)}\right) d x>0 .
$$

By the intermediate value theorem, there exists $t \in(0,1)$ such that $\gamma(t) \in \mathscr{N}_{I}$, which implies $c \leq \inf _{\gamma \in \Gamma} \max _{0 \leq t \leq 1} I(\gamma(t))$. Thus (5.1) holds.

Now, in view of Theorem 4.4,c>0. Moreover there exists $\left\{u_{n}\right\} \subset W_{0}^{1, p(\cdot)}(\Omega)$ such that $I\left(u_{n}\right) \rightarrow c$ and $I^{\prime}\left(u_{n}\right) \rightarrow 0$ in $\left(W_{0}^{1, p(\cdot)}(\Omega)\right)^{\prime}$. By Proposition 4.2 and $c>0$, we find a constant $C>0$ such that

$$
\frac{1}{C} \leq \int_{\Omega}\left|\nabla u_{n}(x)\right|^{p(x)} d x \leq C \quad \text { for large } n \in \mathbf{N} .
$$

Here we may assume that $\left\{u_{n}\right\}$ converges weakly to some $u \in W_{0}^{1, p(\cdot)}(\Omega)$; further $\left\{u_{n}(x)\right\}$ and $\left\{\nabla u_{n}(x)\right\}$ converge to $u(x)$ and $\nabla u(x)$ for almost every $x \in \Omega$, respectively. Then it follows that $I^{\prime}(u)=0$. If we show that $u \neq 0$, then $u$ is a nontrivial nonnegative weak solution of (1.3).

On the contrary, suppose $u=0$. Since $I\left(u_{n}\right) \rightarrow c>0$ and $\left\langle I^{\prime}\left(u_{n}\right), u_{n}\right\rangle \rightarrow 0$, taking a subsequence if necessary, we may assume $u_{n}^{+} \neq 0$ for all $n \in \mathbf{N}$. Then for each $n \in \mathbf{N}$, there exists a unique $t_{n} \in(0, \infty)$ such that

$$
\int_{\Omega}\left|\nabla\left(t_{n} u_{n}(x)\right)\right|^{p(x)} d x=\int_{\Omega}\left(t_{n} u_{n}^{+}(x)\right)^{p_{1}^{\sharp}(x)} d x
$$


i.e., $t_{n} u_{n} \in \mathscr{N}_{J}$. We will show $t_{n} \leq 1+o(1)$. On the contrary, if there exists $\varepsilon>0$ such that $t_{n} \geq 1+\varepsilon$ for all $n \in \mathbf{N}$, then

$$
\begin{aligned}
t_{n}^{p^{*}} \int_{\Omega}\left|\nabla u_{n}(x)\right|^{p(x)} d x & \geq \int_{\Omega}\left|\nabla\left(t_{n} u_{n}(x)\right)\right|^{p(x)} d x \\
& =\int_{\Omega}\left(t_{n} u_{n}^{+}(x)\right)^{p_{1}^{\sharp}(x)} d x \geq t_{n}^{q_{*}} \int_{\Omega} u_{n}^{+}(x)^{p_{1}^{\sharp}(x)} d x
\end{aligned}
$$

for all $n \in \mathbf{N}$. Using Lebesgue's convergence theorem, we have

$$
\begin{aligned}
\int_{\Omega}\left|\nabla u_{n}(x)\right|^{p(x)} d x & =\int_{\Omega} u_{n}^{+}(x)^{q(x)} d x+o(1) \\
& =\int_{\left\{x \in \Omega: u_{n}(x) \leq 1\right\}} u_{n}^{+}(x)^{q(x)} d x+\int_{\left\{x \in \Omega: u_{n}(x)>1\right\}} u_{n}^{+}(x)^{q(x)} d x+o(1) \\
& \leq \int_{\Omega} \min \left\{u_{n}^{+}(x), 1\right\} d x+\int_{\Omega} u_{n}^{+}(x)^{p_{1}^{\sharp}(x)} d x+o(1) \\
& \leq \int_{\Omega} u_{n}^{+}(x)^{p_{1}^{\sharp}(x)} d x+o(1) .
\end{aligned}
$$

Hence it follows that

$$
\begin{aligned}
\int_{\Omega}\left|\nabla u_{n}(x)\right|^{p(x)} d x & \geq t_{n}^{q_{*}-p^{*}} \int_{\Omega} u_{n}^{+}(x)^{p_{1}^{\sharp}(x)} d x \geq(1+\varepsilon)^{q_{*}-p^{*}} \int_{\Omega} u_{n}^{+}(x)^{p_{1}^{\sharp}(x)} d x \\
& \geq(1+\varepsilon)^{q_{*}-p^{*}}\left(\int_{\Omega}\left|\nabla u_{n}(x)\right|^{p(x)} d x+o(1)\right)
\end{aligned}
$$

which together with (5.2) yields a contradiction. Thus we have shown $t_{n} \leq 1+o(1)$. On the other hand, for each $n \in \mathbf{N}$, take a unique number $s_{n}>0$ such that

$$
\int_{\Omega}\left|\nabla\left(s_{n} u_{n}(x)\right)\right|^{p(x)} d x=\int_{\Omega}\left(s_{n} u_{n}^{+}(x)\right)^{q(x)} d x,
$$

i.e., $s_{n} u_{n} \in \mathscr{N}_{I}$. We see easily that $I\left(s_{n} u_{n}\right)=\max _{s>0} I\left(s u_{n}\right)$ for each $n \in \mathbf{N}$. By (5.2), (5.3) and $\left\langle I^{\prime}\left(u_{n}\right), u_{n}\right\rangle=o(1)$, we infer that $s_{n}=1+o(1)$, so that

$$
I\left(u_{n}\right)=I\left(s_{n} u_{n}\right)+o(1)=\max _{s \geq 0} I\left(s u_{n}\right)+o(1) \geq I\left(t_{n} u_{n}\right)+o(1) .
$$

Let $\varepsilon \in(0,1)$. Then, noting

$$
\begin{aligned}
\int_{\left\{x \in \Omega: q(x) \leq p_{1}^{\sharp}(x)-\varepsilon\right\}}\left(t_{n} u_{n}^{+}(x)\right)^{q(x)} d x & \leq \int_{\Omega} \min \left\{t_{n} u_{n}^{+}(x), 1\right\} d x+\int_{\Omega}\left(t_{n} u_{n}^{+}(x)\right)^{p_{1}^{\sharp}(x)-\varepsilon} d x \\
& =o(1),
\end{aligned}
$$

we obtain

$$
\begin{aligned}
c & =I\left(u_{n}\right)+o(1) \geq I\left(t_{n} u_{n}\right)+o(1) \\
& \geq \int_{\Omega}\left(\frac{1}{p(x)}\left|\nabla\left(t_{n} u_{n}(x)\right)\right|^{p(x)}-\frac{1}{p_{1}^{\sharp}(x)-\varepsilon}\left(t_{n} u_{n}^{+}(x)\right)^{p_{1}^{\sharp}(x)}\right) d x+o(1) \\
& =J\left(t_{n} u_{n}\right)+\int_{\Omega}\left(\frac{1}{p_{1}^{\sharp}(x)}-\frac{1}{p_{1}^{\sharp}(x)-\varepsilon}\right)\left(t_{n} u_{n}^{+}(x)\right)^{p_{1}^{\sharp}(x)} d x+o(1) \geq \inf _{v \in \mathscr{N}_{J}} J(v)-C \varepsilon,
\end{aligned}
$$

where $C$ is a constant which is independent of $\varepsilon \in(0,1)$. Since $\varepsilon \in(0,1)$ is arbitrary, we conclude that $c \geq \inf _{v \in \mathscr{N}_{J}} J(v)$, which contradicts our assumption. Hence it follows that $u \neq 0$, as required. 
We denote by $\mathscr{D}^{1, p(\cdot)}\left(\mathbf{R}^{N}\right)$ the completion of $C_{0}^{\infty}\left(\mathbf{R}^{N}\right)$ by the norm $\|\nabla u\|_{L^{p(\cdot)}\left(\mathbf{R}^{N}\right)}$ in $C_{0}^{\infty}\left(\mathbf{R}^{N}\right)$.

Theorem 5.2. Let $p(\cdot): \mathbf{R}^{N} \rightarrow \mathbf{R}$ be a log-Hölder continuous function with $1<p_{*} \leq p^{*}<N$, and let $q(\cdot): \mathbf{R}^{N} \rightarrow \mathbf{R}$ be a measurable function such that $p^{*}<q_{*} \leq q(x) \leq p_{1}^{\sharp}(x)$ for almost every $x \in \mathbf{R}^{N}$. Assume that $\mathscr{D}^{1, p(\cdot)}\left(\mathbf{R}^{N}\right)$ is continuously embedded into $L^{p_{1}^{\sharp}(\cdot)}\left(\mathbf{R}^{N}\right)$, i.e., there exists a constant $C>0$ such that

$$
\|u\|_{L^{p_{1}^{\sharp}(\cdot)}\left(\mathbf{R}^{N}\right)} \leq C\|\nabla u\|_{L^{p(\cdot)}\left(\mathbf{R}^{N}\right)} \text { for all } u \in \mathscr{D}^{1, p(\cdot)}\left(\mathbf{R}^{N}\right) .
$$

Assume also that there exist a measurable subset $D$ of $\mathbf{R}^{N}$ and a number $q_{0}$ such that

$$
\varlimsup_{R \rightarrow \infty}\left|\left\{x \in B_{1}(0): R x \in D\right\}\right|<\left|B_{1}(0)\right|,
$$

$N \underline{p} /\left(N+p_{*}-\underline{p}\right)<q_{0}<N \underline{p} /(N-\underline{p})$, and $\operatorname{ess}^{\sup _{x \in \mathbf{R}^{N} \backslash D}} q(x) \leq q_{0}$, where $\underline{p}=$ $\underline{\lim }_{|x| \rightarrow \infty} p(x)$. Then there exists $R>0$ such that for each bounded open set $\Omega$ in $\mathbf{R}^{N}$ which contains $B_{R}(0)$, problem (1.3) has a nontrivial nonnegative weak solution.

Proof. We set

$$
\begin{aligned}
J_{\mathbf{R}^{N}}(u) & =\int_{\mathbf{R}^{N}}\left(\frac{1}{p(x)}|\nabla u(x)|^{p(x)}-\frac{1}{p_{1}^{\sharp}(x)} u^{+}(x)^{p_{1}^{\sharp}(x)}\right) d x \quad \text { for } u \in \mathscr{D}^{1, p(\cdot)}\left(\mathbf{R}^{N}\right), \\
\mathscr{N}_{J_{\mathbf{R}^{N}}} & =\left\{u \in \mathscr{D}^{1, p(\cdot)}\left(\mathbf{R}^{N}\right) \backslash\{0\}: \int_{\mathbf{R}^{N}}|\nabla u(x)|^{p(x)} d x=\int_{\mathbf{R}^{N}} u^{+}(x)^{p_{1}^{\sharp}(x)} d x\right\} .
\end{aligned}
$$

By Lemma 2.1 we have for $u \in \mathscr{N}_{J_{\mathbf{R}^{N}}}$

$$
\begin{aligned}
& \min \left\{\|\nabla u\|_{L^{p(\cdot)}\left(\mathbf{R}^{N}\right)}^{p_{*}},\|\nabla u\|_{L^{p(\cdot)}\left(\mathbf{R}^{N}\right)}^{p^{*}}\right\} \leq \int_{\mathbf{R}^{N}}|\nabla u(x)|^{p(x)} d x \\
& =\int_{\mathbf{R}^{N}} u^{+}(x)^{p_{1}^{\sharp}(x)} d x \leq \max \left\{\left\|u^{+}\right\|_{L^{p_{1}^{\sharp}(\cdot)}\left(\mathbf{R}^{N}\right)}^{\left(p_{1}^{\sharp}\right)},\left\|u^{+}\right\|_{L^{p_{1}^{\sharp}(\cdot)}\left(\mathbf{R}^{N}\right)}^{\left(p_{1}^{\sharp}\right)^{*}}\right\},
\end{aligned}
$$

which together with (5.4) implies that

$$
\inf _{u \in \mathscr{N}_{J} \mathbf{R}^{N}}\|\nabla u\|_{L^{p(\cdot)}\left(\mathbf{R}^{N}\right)}>0 .
$$

Hence we infer that

$$
\inf _{u \in \mathscr{N}_{\mathbf{R}^{N}}} J_{\mathbf{R}^{N}}(u)>0
$$

Choose any $p_{0}$ such that

$$
1<p_{0}<\underline{p} \text { and } \frac{N p_{0}}{N+p_{*}-p_{0}}<q_{0}<\frac{N p_{0}}{N-p_{0}} .
$$

Let $\bar{u}_{1} \in W_{0}^{1, p_{0}}\left(B_{1}(0)\right)$ be a weak solution of the problem

$$
\left\{\begin{aligned}
-\operatorname{div}\left(|\nabla u(x)|^{p_{0}-2} \nabla u(x)\right) & =u(x)^{q_{0}-1} & & \text { in } B_{1}(0), \\
u(x) & >0 & & \text { in } B_{1}(0), \\
u(x) & =0 & & \text { on } \partial B_{1}(0) .
\end{aligned}\right.
$$

According to [24, Theorem 1] or [33, Proposition 2.1], we see that $\bar{u}_{1} \in C^{1, \beta}\left(\overline{B_{1}(0)}\right)$ for some $\beta \in(0,1)$. Hence, for each $R>0, \bar{u}_{R}(x) \equiv R^{-p_{0} /\left(q_{0}-p_{0}\right)} \bar{u}_{1}(x / R)$ is a weak 
solution of (5.7). Take $R_{1}>0$ such that $\max _{|x| \leq R} \bar{u}_{R}(x) \leq 1$ for $R \geq R_{1}$. For each $R>0$, there exists a unique $t_{R} \in(0, \infty)$ such that

$$
\int_{B_{R}(0)}\left|\nabla\left(t_{R} \bar{u}_{R}(x)\right)\right|^{p(x)} d x=\int_{B_{R}(0)}\left|t_{R} \bar{u}_{R}(x)\right|^{q(x)} d x .
$$

From (5.5), we find $\delta>0$ and $R_{2} \geq R_{1}$ such that

$$
\left|\left\{x \in B_{1}(0): R x \in D\right\}\right| \leq\left|B_{1}(0)\right|-\delta \quad \text { for each } R \geq R_{2} .
$$

We will show $\left\{t_{R}: R \geq R_{2}\right\}$ is bounded. If $t_{R}>1$ with $R \geq R_{2}$, then we have

$$
\begin{aligned}
t_{R}^{p^{*}} \int_{B_{R}(0)}\left|\nabla \bar{u}_{R}(x)\right|^{p(x)} d x & \geq \int_{B_{R}(0)}\left|t_{R} \bar{u}_{R}(x)\right|^{q(x)} d x \geq t_{R}^{q_{*}} \int_{B_{R}(0) \backslash D}\left|\bar{u}_{R}(x)\right|^{q_{0}} d x \\
& =t_{R}^{q_{*}}\left(\int_{B_{R}(0)}\left|\bar{u}_{R}(x)\right|^{q_{0}} d x-\int_{B_{R}(0) \cap D}\left|\bar{u}_{R}(x)\right|^{q_{0}} d x\right),
\end{aligned}
$$

which implies

$$
t_{R}^{q_{*}-p^{*}} \leq \frac{\int_{B_{1}(0)} R^{\frac{q_{0}\left(p_{0}-p(R x)\right)}{q_{0}-p_{0}}}\left|\nabla \bar{u}_{1}(x)\right|^{p(R x)} d x}{\int_{B_{1}(0)}\left|\bar{u}_{1}(x)\right|^{q_{0}} d x-\sup \left\{\int_{A}\left|\bar{u}_{1}(x)\right|^{q_{0}} d x: A \subset B_{1}(0),|A| \leq\left|B_{1}(0)\right|-\delta\right\}} .
$$

Let $r_{0}>0$ such that $p(x)>p_{0}$ for all $x \in \mathbf{R}^{N}$ with $|x| \geq r_{0}$. By (5.6) and the boundedness of $\left|\nabla \bar{u}_{1}\right|$, we have for $R \geq r_{0}$,

$$
\begin{aligned}
\int_{B_{1}(0)} R^{\frac{q_{0}\left(p_{0}-p(R x)\right)}{q_{0}-p_{0}}}\left|\nabla \bar{u}_{1}(x)\right|^{p(R x)} d x & \leq C\left(\int_{|x|<r_{0} / R} R^{\frac{q_{0}\left(p_{0}-p(R x)\right)}{q_{0}-p_{0}}} d x\right. \\
\left.+\int_{r_{0} / R \leq|x| \leq 1} R^{\frac{q_{0}\left(p_{0}-p(R x)\right)}{q_{0}-p_{0}}} d x\right) & \leq C\left(R^{\frac{q_{0}\left(p_{0}-p_{*}\right)}{q_{0}-p_{0}}}\left(\frac{r_{0}}{R}\right)^{N}+1\right) \leq C,
\end{aligned}
$$

where each $C$ is a positive constant which is independent of $R$. Hence we insist that $\left\{t_{R}: R \geq R_{2}\right\}$ is bounded. Then we have

$$
\begin{aligned}
& \int_{B_{R}(0)}\left(\frac{1}{p(x)}\left|\nabla\left(t_{R} \bar{u}_{R}(x)\right)\right|^{p(x)}-\frac{1}{q(x)}\left|t_{R} \bar{u}_{R}(x)\right|^{q(x)}\right) d x \leq C \int_{B_{R}(0)}\left|\nabla \bar{u}_{R}(x)\right|^{p(x)} d x \\
& =C \int_{B_{1}(0)} R^{-\frac{q_{0} p(R x)}{q_{0}-p_{0}}+N}\left|\nabla \bar{u}_{1}(x)\right|^{p(R x)} d x \leq C\left(R^{-\frac{q_{0} p_{*}}{q_{0}-p_{0}}} r_{0}^{N}+R^{-\frac{q_{0} p_{0}}{q_{0}-p_{0}}+N}\right) \rightarrow 0
\end{aligned}
$$

as $R \rightarrow \infty$. Hence we can find $R \geq R_{2}$ satisfying

$$
\int_{B_{R}(0)}\left(\frac{1}{p(x)}\left|\nabla\left(t_{R} \bar{u}_{R}(x)\right)\right|^{p(x)}-\frac{1}{q(x)}\left|t_{R} \bar{u}_{R}(x)\right|^{q(x)}\right) d x<\inf _{v \in \mathscr{N}_{\mathbf{R}^{N}}} J_{\mathbf{R}^{N}}(v) .
$$

Now, let $\Omega$ be any bounded open set which contains $B_{R}(0)$. Extending $\bar{u}_{R}$ on $\Omega$ with $\bar{u}_{R}(x)=0$ for $x \in \Omega \backslash B_{R}(0)$, we have $\bar{u}_{R} \in W_{0}^{1, p(\cdot)}(\Omega)$. Letting $I, J, \mathscr{N}_{I}$ and $\mathscr{N}_{J}$ be as in the previous proposition, we have

$$
\inf _{v \in \mathscr{N}_{I}} I(v) \leq I\left(t_{R} \bar{u}_{R}\right)<\inf _{v \in \mathscr{N}_{\mathbf{R}^{N}}} J_{\mathbf{R}^{N}}(v) \leq \inf _{v \in \mathscr{N}_{J}} J(v) .
$$

Hence problem (1.3) has a nontrivial nonnegative weak solution on $\Omega$ by the proposition.

Finally, we give a sufficient condition for (5.4). We recall the following result, which is a special case of [6, Theorem 1.8]. 
Lemma 5.3. Let $p(\cdot): \mathbf{R}^{N} \rightarrow \mathbf{R}$ be a log-Hölder continuous function which satisfies $1<p_{*} \leq p^{*}<N$ and

$$
|p(x)-p(y)| \leq \frac{C}{\log (e+|x|)} \quad \text { for each } x, y \in \mathbf{R}^{N} \text { with }|y| \geq|x| .
$$

Then the fractional integral operator

$$
u \mapsto \int_{\mathbf{R}^{N}} \frac{u(y)}{|x-y|^{N-1}} d y
$$

is bounded from $L^{p(\cdot)}\left(\mathbf{R}^{N}\right)$ to $L^{p_{1}^{\sharp}(\cdot)}\left(\mathbf{R}^{N}\right)$.

Corollary 5.4. Let $p(\cdot): \mathbf{R}^{N} \rightarrow \mathbf{R}$ be as in the previous lemma, and let $D, q_{0}$ and $q(\cdot)$ be as in Theorem 5.2. Then there exists $R>0$ such that for each bounded open set $\Omega$ in $\mathbf{R}^{N}$ which contains $B_{R}(0)$, problem (1.3) has a nontrivial nonnegative weak solution.

Proof. Using the previous lemma, we can show that $\mathscr{D}^{1, p(\cdot)}\left(\mathbf{R}^{N}\right)$ is continuously embedded into $L^{p^{\sharp}(\cdot)}\left(\mathbf{R}^{N}\right)$ by similar lines as those in [35, p. 88]. Hence we obtain the conclusion by Theorem 5.2.

\section{References}

[1] Adams, R. A., and J. J. F. Fournier: Sobolev spaces. - Academic Press, 2003.

[2] Alves, C. O., and M. A. S. Souto: Existence of solutions for a class of problems in $\mathbf{R}^{N}$ involving the $p(x)$-Laplacian. - In: Contribution to Nonlinear Analysis, Progr. Nonlinear Differential Equations Appl. 66, 2006, 17-32.

[3] BAhri, A., and M. Coron: On a nonlinear elliptic equation involving the critical Sobolev exponent: the effect of the topology of the domain. - Comm. Pure Appl. Math. 41, 1988, 253-294.

[4] Boccardo, L., and F. Murat: Almost everywhere convergence of the gradients of solutions to elliptic and parabolic equations. - Nonlinear Anal. 19, 1992, 581-597.

[5] Boureanu, M.-M.: Existence of solutions for an elliptic equation involving the $p(x)$-Laplace operator. - Electron. J. Differential Equations 2006:97, 2006, 1-10.

[6] Capone, C., D. Cruz-Uribe, SFO, and A. Fiorenza: The fractional maximal operators on variable $L^{p}$ spaces. - Rev. Mat. Iberoamericana 23:3, 2007, 743-770.

[7] Coron, J.: Topologie et cas limite des injections de Sobolev. - C. R. Acad. Sci. Paris Sér. I Math. 299, 1984, 209-212.

[8] Diening, L.: Riesz potential and Sobolev embeddings on generalized Lebesgue and Sobolev spaces $L^{p(\cdot)}$ and $W^{k, p(\cdot)}$. - Math. Nachr. 268, 2004, 31-43.

[9] DinU, T.-L.: Entire solutions of multivalued nonlinear Schrödinger equations in Sobolev spaces with variable exponent. - Nonlinear Anal. 65, 2006, 1414-1424.

[10] Edmunds, D. E., and J. RÁKosník: Sobolev embeddings with variable exponent. - Studia Math. 143, 2000, 267-293.

[11] FAn, X.: Solutions for $p(x)$-Laplacian Dirichlet problems with singular coefficients. - J. Math. Anal. Appl. 312, 2005, 464-477.

[12] FAN, X., and C. JI: Existence of infinitely many solutions for a Neumann problem involving the $p(x)$-Laplacian. - J. Math. Anal. Appl. 334, 2007, 248-260.

[13] FAn, X., and X. HAN: Existence and multiplicity of solutions for $p(x)$-Laplacian equations in $\mathbf{R}^{N}$. - Nonlinear Anal. 59, 2004, 173-188.

[14] Fan, X., J. Shen, and D. ZhaO: Sobolev embedding theorems for spaces $W^{k, p(x)}(\Omega)$. - J. Math. Anal. Appl. 262, 2001, 749-760. 
[15] FAn, X., and G.-H. Zhang: Existence of solutions for $p(x)$-Laplacian Dirichlet problem. Nonlinear Anal. 52, 2003, 1843-1852.

[16] FAn, X., and D. ZhaO: A class of De Giorgi type and Hölder continuity. - Nonlinear Anal. $36,1999,295-318$.

[17] FAn, X., and D. ZhaO: On the spaces $L^{p(x)}(\Omega)$ and $W^{m, p(x)}(\Omega)$. - J. Math. Anal. Appl. 263, $2001,424-446$.

[18] Futamura, T., Y. Mizuta, and T. Shimomura: Sobolev embeddings for Riesz potential space of variable exponent. - Math. Nachr. 279, 2006, 1463-1473.

[19] Futamura, T., Y. Mizuta, and T. Shimomura: Sobolev embeddings for variable exponent Riesz potentials on metric spaces. - Ann. Acad. Sci. Fenn. Math. 31, 2006, 495-522.

[20] Harjulehto, P., and P. Hästö: Sobolev inequalities for variable exponents attaining the values 1 and $n$. - Publ. Mat. 52:2, 2008, 347-363.

[21] Harjulehto, P., P. Hästö, M. Koskenoja, and S. Varonen: The Dirichlet energy integral and variable exponent Sobolev spaces with zero boundary values. - Potential Anal. 25, 2006, 205-222.

[22] Kovácik, O., and J. RÁkosník: On spaces $L^{p(x)}$ and $W^{k, p(x)}$. - Czechoslovak Math. J. 41, 1991, 592-618.

[23] Kurata, K., and N. ShioJi: Compact embedding from $W_{0}^{1,2}(\Omega)$ to $L^{q(x)}(\Omega)$ and its application to nonlinear elliptic boundary value problem with variable critical exponent. - J. Math. Anal. Appl. 339, 2008, 1386-1394.

[24] Lieberman, G. M.: Boundary regularity for solutions of degenerate elliptic equations. - Nonlinear Anal. 12, 1988, 1203-1219.

[25] Mattila, P.: Geometry of sets and measures in Euclidean spaces. - Cambridge Univ. Press, 1995.

[26] Mizuta, Y., T. Ohno, and T. Shimomura: Integrability of maximal functions for generalized Lebesgue spaces with variable exponent. - Math. Nachr. 281, 2008, 386-395.

[27] Mizuta, Y., T. Ohno, and T. Shimomura: Sobolev's inequalities and vanishing integrability for Riesz potentials of functions in the generalized Lebesgue space $L^{p(\cdot)}(\log L)^{q(\cdot)}$. - J. Math. Anal. Appl. (to appear).

[28] Mizuta, Y., and T. Shimomura: Sobolev's inequality for Riesz potentials with variable exponent satisfying a log-Hölder condition at infinity. - J. Math. Anal. Appl. 311, 2005, 268288.

[29] Orlicz, W.: Über konjugierte Exponentenfolgen. - Studia Math. 3, 1931, 200-211.

[30] Passaseo, D.: Multiplicity of positive solutions of nonlinear elliptic equations with critical Sobolev exponent in some contractile domains. - Manuscripta Math. 65:2, 1989, 147-165.

[31] Pohozaev, S.: Eigenfunctions of the equation $\Delta u+\lambda f(u)=0$. - Soviet Math. Dokl. 6, 1965, 1408-1411.

[32] RŮŽIČKA, M.: Electrorheological fluids: modeling and mathematical theory. - Lecture Notes in Math. 1748, Springer, 2000.

[33] TAKÁČ, P.: Nonlinear spectral problems for degenerate elliptic operators. - In: Stationary partial differential equations, Vol. I, Handb. Differ. Equ., North-Holland, Amsterdam, 2004, $385-489$.

[34] Willem, M.: Minimax theorems. - Progr. Nonlinear Differential Equations Appl. 24, Birkhäuser Boston, Inc., Boston, MA, 1996.

[35] Ziemer, W. P.: Weakly differentiable functions. - Grad. Texts in Math. 120, Springer-Verlag, Berlin, 1989. 